ENCYCLOPEDDIE Encyclopédie berbère

BERBERE

32 | 2010

32 | Mgild - Mzab

\title{
Moullon (à manchettes)
}

N. Manlius

\section{OpenEdition}

Journals

Édition électronique

URL : https://journals.openedition.org/encyclopedieberbere/629

DOI : $10.4000 /$ encyclopedieberbere.629

ISSN : 2262-7197

\section{Éditeur}

Peeters Publishers

\section{Édition imprimée}

Date de publication : 31 décembre 2010

Pagination : 5077-5082

ISBN : 978-90-429-2369-0

ISSN : 1015-7344

\section{Référence électronique}

N. Manlius, « Moullon (à manchettes) », Encyclopédie berbère [En ligne], 32 | 2010, document M130a, mis en ligne le 11 novembre 2020, consulté le 17 février 2022. URL : http://journals.openedition.org/ encyclopedieberbere/629; DOI : https://doi.org/10.4000/encyclopedieberbere.629

Ce document a été généré automatiquement le 17 février 2022.

(c) Tous droits réservés 


\title{
Moullon (à manchettes)
}

\author{
N. Manlius
}

1 Appelé en berbère udad, plur. udaden (touareg, chleuh...), le mouflon à manchettes appartient à l'ordre des Artiodactyles, famille des Bovidés, sous-famille des Caprinés, et constitue la seule espèce actuelle du genre Ammotragus. En effet sa position phylogénétique est incertaine. Son genre est encore considéré par certains auteurs comme pouvant être intermédiaire entre celui de deux autres Caprinés, les caprins (genre Capra) et les ovins (genre Ovis), tandis que pour d'autres paléontologues, cette lignée est considérée comme proche des deux autres lignées de Caprinés, les caprins (genre Capra) et les ovins (genre Ovis). Sa ressemblance morphologique avec les mouflons eurasiatiques est toutefois à l'origine du nom de "mouflon à manchettes » donné par les premiers voyageurs européens dans le nord de l'Afrique.

Pelage homochrome roux à isabelle, femelles et mâles atteignent respectivement jusqu'à $0,90 \mathrm{~m}$ et $1 \mathrm{~m}$ au garrot pour une longueur moyenne (avec queue) de $1,35 \mathrm{~m}$ et $1,60 \mathrm{~m}$. Les fourchettes de poids sont respectivement de 40-65 kg et 100-145 kg. Les vieux mâles se distinguent par des cornes enroulées vers l'extérieur et vers l'arrière pour revenir sur le devant à la pointe, ainsi que par une longue crinière claire touchant presque le sol et débutant à la base du menton pour s'étendre au cou, à l'ensemble du poitrail et sur le haut des membres antérieurs, dont elle finit par entourer les coudes à la manière de manchettes.

3 Excellent grimpeur, remarquable sauteur, il affectionne les régions montagneuses jusqu'à des altitudes avoisinant les $3000 \mathrm{~m}$. Lorsqu'il partage son habitat avec le bouquetin, il fréquente plutôt les strates inférieures. Animal très bien adapté à la vie dans le désert, sa physiologie lui permet de vivre plusieurs années sans boire en se contentant de l'eau des plantes. Il adopte alors un comportement en rapport : mode de vie en groupes familiaux, activité tôt le matin et tard le soir. Le record de longévité, mesuré en captivité, est de 24 ans (Haltenorth et Diller 1985). 


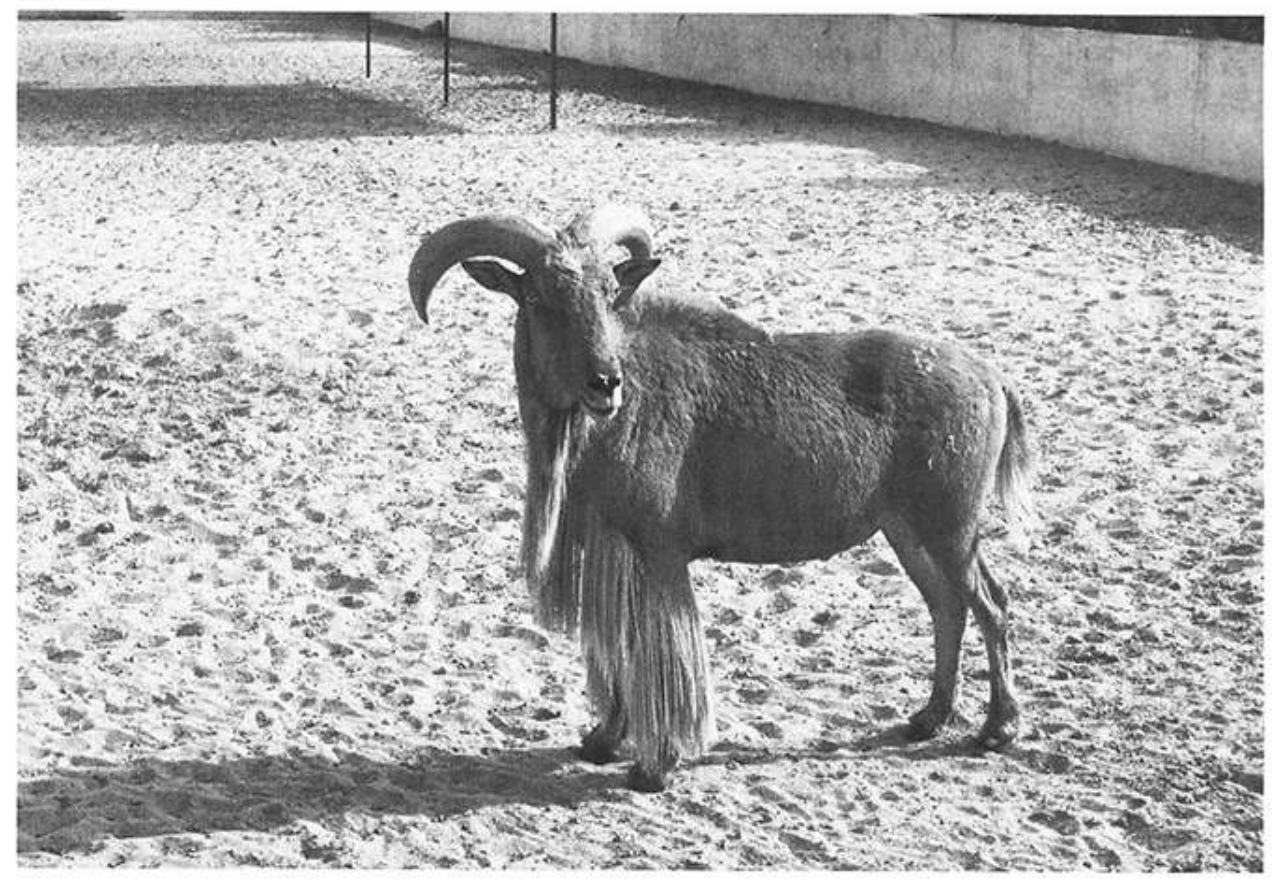

FIg. 1. DeUX Femelles MOUfLON À MANCHETtes ACcompagnées d'Un JeUNe. PHOTOgRAPHIE PRISE PAR L'AUTEUR AU JARDIN ZOOLOgIQUE DE GIZEH, AVRIL 1999.

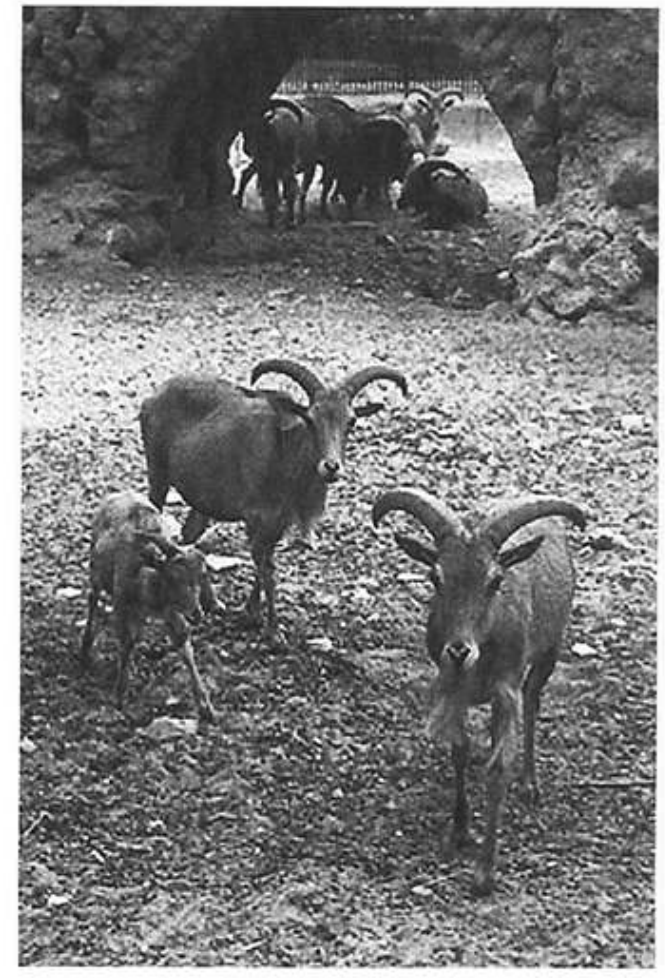

Fig. 2. Un MÂLE MOUfLON À MANCHETTES. PHOTOgRAPHIE PRISE PAR L'AUTEUR AU JARDIN ZOOLOgIQUE DE GIZEH, AVRIL 1999.

4 Il y a peu, on croyait le genre Ammotragus endémique à l'Afrique, mais des restes d'une nouvelle espèce, Ammotragus europaeus, datés d'un million d'années, ont récemment étés découverts dans le sud-ouest de l'Europe (Moullé et al. 2004). 
5 Les hommes de la culture ibéromaurusienne (vivant au Maghreb entre 22000 et 8000 av. J.-C.) marquèrent une nette préférence pour sa chasse car sa viande était déjà appréciée. Certains se firent même inhumer avec les cornes d'un mouflon à manchettes (Merzoug 2005; Hachi 2006). Au Maroc, il était l'espèce dominante dans les niveaux ibéromaurusiens de Kaf Taht-El-Ghar (Ouchaou et Amani 1997), d'Ifri N'Ammar (Mikdad et Eiwanger 2000) et du Kehf el-Hammar (Barton et al. 2005).

De récentes fouilles effectuées dans le massif Acacus, dans le sud-ouest de la Libye, tendraient à montrer qu'au début de l'Holocène (vers 7000 ans av. J.-C.) des populations de chasseurs-cueilleurs (probablement en relation avec les peintures "Têtes rondes») auraient maintenu de jeunes adultes en captivité pour disposer d'une réserve de nourriture fraîche immédiatement disponible. Ils n'en auraient cependant pas fait l'élevage sensu stricto (Di Lernia 2001). Il est d'ailleurs plus que probable que le mouflon à manchettes ne fut jamais un des ancêtres du mouton domestique actuel.

7 Ainsi, les premiers élevages de caprins et d'ovins du Maghreb oriental, pratiqués entre 5906-4910 cal BC et 3367-2828 cal BC, notamment dans la grotte Capéletti de l'Aurès en Algérie (Roubet 2003, et Roubet in EB, XXVII, 2005), n'auraient pas pour origine des populations locales de mouflons à manchettes. Avec la Néolithisation, il semblerait que celui-ci ait progressivement perdu, même en région montagneuse, son statut d'animal de bouche préféré et d'emblème culturel (ibéromaurusien) au profit de caprins et d'ovins domestiques. En particulier, le bélier adulte devint le nouvel emblème culturel : élevé au rang d'animal-culte, on le voit porteur d'ornements de parade sur les gravures rupestres de l'Atlas saharien en Algérie (Roubet 2003).

De nos jours, l'aire de distribution du mouflon à manchettes est fragmentée en îlots de taille variable dans l'ensemble des pays du Sahara. Ses effectifs les plus importants sont concentrés dans les pays du Maghreb : il est encore présent, quoiqu'en danger, dans les montagnes du Maroc (Panouze 1957 ; Aulagnier et Thévenot 1997, 2006), de l'Algérie, au nord et au sud (de Smet 1997a), et de la Tunisie (de Smet 1997b). Son aire s'étend vers l'est jusqu'en Egypte dans le Djebel Ouénat (Manlius et al. 2003 ; Menardi-Noguera 2008 (comm. pers.) ; Zboray, 2008 (comm. pers.) et près de la Mer Rouge dans le Djebel Elba (Wacher et al. 2002). Bien qu'il n'existe aucune preuves (ostéologiques, iconographiques ou épigraphiques) formelles à ce sujet, à l'exception d'informations précisant que durant les années 1700 son aire de distribution atteignait les montagnes à l'est du Caire non loin de l'isthme de Suez, il ne serait pas impossible que, durant l'Holocène ou même au début de la période historique, le mouflon à manchettes ait pu vivre dans la péninsule du Sinaï, voire même au Proche-Orient.

9 Certes, il continue de se maintenir dans certaines réserves bien protégées, notamment en Tunisie (de Smet 1997b), mais la chasse très soutenue dont il continue de faire l'objet le met particulièrement en danger dans de nombreuses autres régions. Ainsi, alors qu'il y a peu il fréquentait encore tous les reliefs de l'Afrique du Nord (en 1950, il occupait la totalité des zones montagneuses marocaines), il ne se rencontre plus de nos jours que dans quelques zones de l'Atlas. Les menaces sont en effet bien réelles: l'homme tout d'abord, premièrement avec la chasse (piégeage par les habitants du désert, tir à vu par des braconniers ou des militaires, massacres organisés par de riches particuliers), et secondement du fait de déforestations ou de surpâturages par les troupeaux domestiques; les animaux ensuite, essentiellement les chiens sauvages (Aulagnier 1992); le climat enfin (il arrive que des périodes de grandes sécheresse déciment des troupeaux entiers). 

Etats-Unis durant les années 1950 (Gray et Simpson 1980), sa totale réussite adaptative (plus de 10.000 individus en 1985) y menace maintenant le mouflon local (Cassinello 1998). De là, il s'est répandu ou a été relâché au Mexique et gagne maintenant le centre de ce pays (Álvarez-Romero et Medellín 2005). De la même manière, introduit en 1970 dans le sud-est de l'Espagne, il s'y développa avec une aisance telle qu'il nuit désormais au bouquetin local (Cassinello 2000; Acevedo et al. 2007). Enfin, introduit dans l'île canarienne de La Palma en 1972, il y menace maintenant gravement la flore endémique (Cassinello 1998). On voit donc que, soustrait à une pression de chasse d'autant plus forte qu'elle s'exerce en milieu hyperaride, le mouflon à manchettes se révèle être une espèce particulièrement vigoureuse et conquérante.

C'est peut-être à cette étonnante réussite dans les régions d'introduction qu'est dû son non moins étonnant classement parmi (seulement) les espèces vulnérables sur la liste rouge de l'UICN des espèces menacées (IUCN 2007).

\section{BIBLIOGRAPHIE}

ACEVEDO, P. et al., 2007 - "Invasive exotic aoudad (Ammotragus lervia) as a major threat to native Iberian ibex (Capra pyrenaica) : a habitat suitability model approach". Diversity \& Distributions, 13 (5) p. 587-597.

ALVAREZ-ROMERo J. \& MEDELLÍN R. A., 2005 - Ammotragus lervia. Vertebrados superiores exóticos en México : diversidad, distribución y efectos potenciales. Instituto de Ecología, Universidad Nacional Autónoma de México. Bases de datos SNIB-CoNABIO. Proyecto U020. México. D.F.

AULAGNIER S., 1992 - Zoogéographie des Mammifères du Maroc : de l'analyse spécifique à la typologie de peuplement à l'échelle régionale. Thèse de doctorat d'Etat en Sciences, Université de Montpellier II, EPHE, Montpellier, 235 p.

AULAGNIER S. \& M. THÉVENOT, 1997 - “Morocoo (including Western Sahara)”. In: Wild sheep and goats and their relatives : status survey and conservation action plan for Caprinae. Shackleton D. M. et IUCN/ ssc, Caprinae Specialist Group (éd.), Gland : 34-38.

AULAGNIER S. \& M. THÉVENOT, 2006 - « Mise à jour de la liste des mammifères sauvages du Maroc. Janvier 2006". Go-South Bulletin, $3:$ 6-9.

BARTON R.N.E., BOUZOUGGAR A., COLLCUTT S.N., GALE R., HIGHMAN T.F.G., HUMPHREY L.T., PARFITT S., STRINGER C.B., \& MALEK F., 2005 - "The Late Upper Palaeolithic occupation of the Moroccan Northwest Maghreb during the last glacial maximum”. African Archaeological Review, vol. 22, $\mathrm{n}^{\circ} 2$, p. $77-100$.

CASSINELLO J., 1998 - “Ammotragus lervia : a review on systematics, biology, ecology and distribution”. Annales Zoologici Fennici, 35 (3) : 149-162.

CASSINELLO, J., 2000 - “Ammotragus free-ranging population in the south-east of Spain : a necessary first account”. Biodiversity and Conservation, 9 (7) : 887-900. 
DE SMET K., 1997a - "Algeria". In : Wild sheep and goats and their relatives : status survey and conservation action plan for Caprinae. Shackleton, D. M. et IUCN/SSC Caprinae Specialist Group (éd.), Gland : 17-19.

DE SMET K., 1997b - « Tunisia ». In: Wild sheep and goats and their relatives : status survey and conservation action plan for Caprinae. Shackleton, D. M. et IUCN/SSC Caprinae Specialist Group (éd.), Gland : 45-47.

DI LERNIA S., 2001 - "Dismanting dung : delayed use of food resources among Early Holocene foragers of the Libyan Sahara". Journal of Anthropological Archaeology, 20 (4) : 408-441.

GRAY G. G. \& SIMPSON D., 1980 - “Ammotragus lervia”. Mammalian Species, 144 : 1-7.

HACHI S. 2006 - « Du comportement symbolique des derniers chasseurs Mechta-Afalou d'Afrique du Nord ». Comptes Rendus Palévol, 5 (1-2) : 429-440.

HALTENORTH, T. \& DILLER H., 1985 - « Mammifères d'Afrique et de Madagascar ». Delachaux \& Niestlé, Neuchâtel, 397 p.

IUCN 2007., 2007IUCN Red List of Threatened Species. <www.iucnredlist.org>.

MANLIUS N. \& al., 2003 - "Decline of the Barbary sheep, Ammotragus lervia, in Egypt during the 20th century : literature review and recent observations". Journal of Zoology, 259 (4) : 403-409.

MERZOUG S., 2005 - Comportements de subsistance des Ibéromaurusiens d'apres l'analyse archéozoologique des Mammiferes des sites de Tamar Hat, Taza 1, et Columnata (Algérie). Doctorat du MNHN, Département de Préhistoire, Institut de Paléontologue Humaine, Paris, 414 p.

MIKDAD A. K. \& EIWANGER J., 2000 - „Recherches préhistoriques et protohistoriques dans le Rif oriental (Maroc). Rapport préliminaire ». AVA Beiträge, 20, p. 109-167.

MOULLÉ P.-E. et al., 2004 - « Ammotragus europaeus : une nouvelle espèce de Caprini (Bovidae, Mammalia) du Pléistocène inférieur à la grotte du Vallonnet (France) ». Comptes Rendus Palévol, 3 (8) : 663-673.

OUCHAOU B. \& AMANI F., 1997 - «Etude préliminaire des grands Mammifères du gisement de KafTaht-El-Ghar (Tétouan, Maroc) ». Préhistoire et Anthropologie Méditerranéennes, t.6, p. 53-60.

PANOUSE J.B., 1957 - Les Mammifères du Maroc. Primates, Carnivores, Pinnipèdes, Artiodactyles. Trav. de l'Institut Chérifien, série Zoologie, nº 5, 206 p.

ROUBET C., 2003 - «Statut de Berger » des communautés atlasiques, néolithisées du Maghreb oriental, dès $7000 \mathrm{BP} »$. L'Anthropologie, 107 (3) : 393-442.

WACHER T. et al., 2002 - "New observations of the 'extinct' Aoudad Ammotragus lervia ornata in Egypt". Oryx, 36 (3) : 301-304.

INDEX

Mots-clés : Chasse, Ethnologie, Ethnographie, Préhistoire, Zoologie 